\title{
"KERATOCYSTIC ODONTOGENIC TUMOR OF MANDIBULAR THIRD MOLAR RESEMBLING AN ODONTOME: A CASE REPORT"
}

Shahanavaj. I. Khaji

1. Senior Lecturer,. Department of Oral \& Maxillofacial Surgery, Tatyasaheb Kore Dental College \& Research Centre, Mahatma gandhi hospital campus Maharastra.

\section{CORRESPONDING AUTHOR:}

Dr. Shahanavaj. I.

Khaji, Department of Oral \& Maxillofacial Surgery,

Tatyasaheb Kore Dental College \& Research Centre,

Mahatma gandhi hospital campus,

New Pargaon- 416137. Kolhapur,Maharastra, India.

E-mail: forevershaan@gmail.com

\begin{abstract}
The keratocystic odontogenic tumor (KCOT) is a relatively common oral \& maxillofacial lesion that derives its origin from remnants of dental lamina. [1] KCOT represents a special group because of their aggressive growth \& recurrence. [2]

KCOT's are known for their most aggressive behavior, innate growth potential \& tendency to invade adjacent hard \& soft tissues causing significant morbidity \& mortality.

It has a high recurrence rate and is associated with the basal cell nevus syndrome [3]. We hereby report an asymptomatic case of KCOT associated with floating left mandibular third molar resembling an odontome in a 21 years old male patient which was treated by original conservative method.
\end{abstract}

KEYWORDS: Keratocystic odontogenic tumor, Floating mandibular third molar, KCOT.

INTRODUCTION: KCOT is defined as "a benign uni or multicystic, intraosseous tumor of odontogenic origin, with a characteristic lining of parakeratinized stratified squamous epithelium \& potential for aggressive, infiltrative behavior". [4]

This lesion was first described in 1956 by PHILIPSEN [5] \& is well known for its high recurrence rate. For this reason, extensive research regarding this lesion has been carried out over the last 48 years. [5-8]

The KCOT contributes approximately $11 \%$ of cysts of the jaws \& is most commonly located in the mandibular ramus \& angle. ${ }^{[8]}$ (May vary from $4-16.5 \%$ ).

The KCOT was reclassified \& renamed as 'keratocystic odontogenic tumor' in the W.H.O classification of head \& neck tumors in 2005. [4]

The odontogenic keratocyst (OKC, currently designated by the World Health Organization as a keratocystic odontogenic tumor) is a locally aggressive, cystic jaw lesion with a putative high growth potential and a propensity for recurrence. [9]

The KCOT is an intra-osseous cystic lesion which generally affects the posterior mandible,\& can become quite large due to its rapid growth \& its extension into the adjacent structures, marked by the presence of a keratinized epithelium which makes it identifiable histopathologically. 


\section{CASE REPORT}

Several treatment options for the management of KCOT's have been described in the literature. Nevertheless surgical enucleation (conservative method) remains the mainstay treatment modality which is proved beneficial \& effective in given clinical_cases taking into account the aggressiveness of the lesion with preservation of neighboring structures. [10]

CASE REPORT: A 21years old male patient was referred to our hospital with the complaints of swelling in the posterior region on the left aspect of his mandible associated with numbness of lower lip since 2-3months.

The patient stated that the problem first occurred 2-3 months ago. There was no history of systemic disease, his medical \& family history was unremarkable.

Extra oral examination, moderate swelling was noted over left side of face, it was also seen that mild vestibular tenderness \& obliteration was evident in relation to 37, 38 region intraorally. (Figure1)

Panoramic radiography was performed which revealed a well defined unilocular radiolucent lesion measuring $1.5 \times 1.8 \mathrm{~cm}$ in diameter with scalloping at the periphery, involving 37 , floating mandibular left third molar resembling an odontome, extending up to the angle, ramus of the mandible. No evidence of root resorption was seen with 37, slight expansion of buccal cortex was evident. (Figure 2)

FNAC revealed viscous, cheesy, purulent material suggestive of an odontogenic cystic lesion. (To rule out vascular lesion)

Considering the dimensions of the lesion, relationship with the neighbouring structures, \& age of the patient; simple enucleation with chemical cauterization (Cornoy's solution application) was chosen as the treatment of choice.

Patient was operated under local anesthesia, extended third molar incision extending along the buccal sulcus - 35,36,37,38 regions (Figure 3) posteriorly beyond the external oblique ridge \& ascending ramus.

Mucoperiosteum reflected, bony window created \& cystic lining identified enucleation of the cyst was carried out in toto with removal of the impacted third molar followed by cornoy 's solution application over the defect \& wound closure achieved by primary intention using Vicryl 3-0 suture material. (Polygalactin 910).

Tissue from the excised specimen was subjected to histopathological evaluation \& was reported as keratocystic odontogenic tumor (Figure 4). Patient was followed up periodically once in three months \& post operative radiographs were taken to observe any recurrence of the lesion, regeneration of the bone defect respectively.

DISCUSSION: KCOT is a benign or multicystic, intraosseous tumor of odontogenic origin. Occurs from $1^{\text {st }}$ to $9^{\text {th }}$ decade with a peak in $2^{\text {nd }} \& 3^{\text {rd }}$ decade, as noted in our case age of the patient was 21 years.

Percentage of KCOT's occurring in the mandible ranges from $65-83 \%$ ( more in ascending ramus). ${ }^{[11]} \mathrm{A}$ localized swelling is the most common symptom (until cyst reaches a considerable size produces pressure symptoms). 


\section{CASE REPORT}

Multiple treatments has been proposed \& debated. The challenge lies in minimizing the risk of recurrence \& morbidity of extensive resection. Numerous modalities ranging from decompression to simple enucleation with or without curettage to resection have been employed. [11]

When choosing the treatment procedure it is important to consider the following:

a) Age of the patient

b) Lesion/s size \& its dimensions

c) Localization \& relationship with the surrounding soft tissues

d) Whether it is primary/ recurrent lesion. [1]

According to GUILIANI.M [13] - Simple enucleation has a recurrence rate of 17-56\%. Simple enucleation combined with adjunctive therapy significantly reduces the recurrence rate to 1-8.7\%. [8] In our case cyst enucleation along with application of cornoy's solution was done to avoid recurrence.

Conservative treatment has advantage of preserving anatomical structures $\&$ is applicable to patients an all age groups. Many authors have reported difficulties in enucleation \& curettage of KCOT's with or without cortical perforation due to adherence of keratocysts thin lining to adjacent bone or soft tissues.

If enucleation is chosen as surgical treatment, special attention must be given to the dentate area, tooth removal should be considered if there is any doubt of leaving pathological tissue behind.

The purpose of using cornoy's solution is to provide total elimination of epithelial remnants from the cyst walls which may cause recurrences.

GOSAU et al (2009) [12] stated that enucleation combined with application of cornoy's solution reduced the recurrence rate compared with simple enucleation. Some authors suggested that if inferior alveolar nerve is exposed in the cavity as in the present case, cornoy's solution was used only once for a period of 3-5 minutes to avoid damage. [12]

CONCLUSION: Treatment of KCOT's remains a controversial subject. Many surgeons tend to treat large sized KCOT's with an aggressive method. The OKC has been the subject of much debate over the last 50 years with respect to its origin, its growth, and treatment modalities. Keratocystic odontogenic tumours are known for their peculiar behaviour, varied origin, debated development, unique tendency to recur and due to propensity of KCOT's to grow within medullary bone they have the potential to become extremely large without causing any clinical signs \& symptoms. Thus, it has been the subject of much research over the last 40 years. It was formerly known as odontogenic keratocyst (OKC). OKC received its new title as keratocystic odontogenic tumor (KCOT) by the WHO (2005) in order to suggest its aggressive and recurrent nature. KCOT is a benign intraosseous neoplasm of the jaw. In any given case clinical \& radiographic follow up is mandatory \& essential.

This case report enlightens the importance of simple surgical enucleation combined with chemical cauterization which could be proved to be a reliable tool in the management of KCOT's for selected cases.

ACKNOWLEDGEMENT: The authors wish to thank the patient whose case is reported in the paper for his permission to use the images \& report his clinical details. 


\section{CASE REPORT}

\section{REFERENCES:}

1. Ozkan A, Bayar RG, Altug AH, Sencimen M, Senel B. Management of KCOT with marsupialization, enucleation \& cornoy's application. A case report OHDM, 2012; June, vol 11 no (2):69-73.

2. Lipovec A, Hren IN. Keratocysts in the jaws. Radiol Oncol 2004; 38(3):187-192.

3. Singh M,Gupta KC.Surgical treatment of OKC by enucleation. Contemp Clin Dent.2010; 1(4):263-267.

4. Philipsen H P. Keratocystic Odontogenic Tumor.WHO classification of tumor series. Pathology \& Genetics of H \& N tumors. LYON; IARC press 2005:306-307.

5. Philipsen $\mathrm{H}$, On keratocysts in the jaws. Tandleagebladet.1956; 60:963.

6. Pindborg J, Hansen J. Studies on odontogenic cyst epithelium II clinical \& roentgraphic aspects of OKC's. Acta Pathol ET Microbial Scand 1953; 58:283.

7. Voorsmit RA, Stoelinga PJ. The management of keratocysts. JOMS.1981; 9:228.

8. Stoelinga PJ. Long term follow up on keratocysts treated according to a defined protocol. IJOMS, 2001; 30:14.

9. Li TJ.The OKC: A cyst or a cystic neoplasm? J Dent Res.2011, Feb; 90(2):133-142.

10. Pham H, Lambert S. OKC'S. Review of literature \& presentation of three clinical cases with different surgical approaches. J Dent Que.1989, May; 26:215-221.

11. Asokan GS, Jeelani S, Pratiban J. KCOT- a case report \& review of literature.Indian journal of dental case reports 2012;2(1):87-92.

12. Gosau M, Draenert FG, Muller S, Frerich B, Burgers R. Two modifications in the treatment of keratocystic odontogenic tumors \& the use of carnoys solution: A retrospective study lasting between 2 \& 10 years. Clinical Oral investigations.2009; 14:27-34.

13. Giuliani M, Gross GB, Lajolo C, Bisceglia M. Conservative management of large OKC- a report of a case \& review of literature.JOMS,2006;64:308-316. 


\section{CASE REPORT}

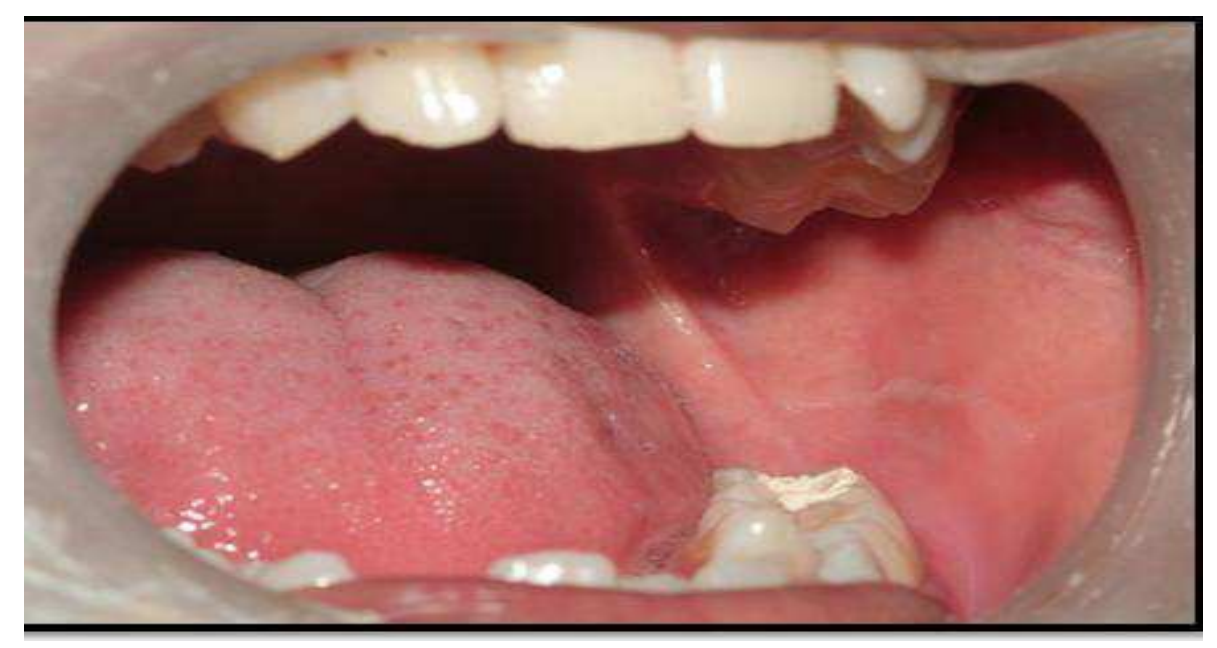

FIGURE 1 - Intra Oral Photograph Shows Vestibular Obliteration 38 Region.

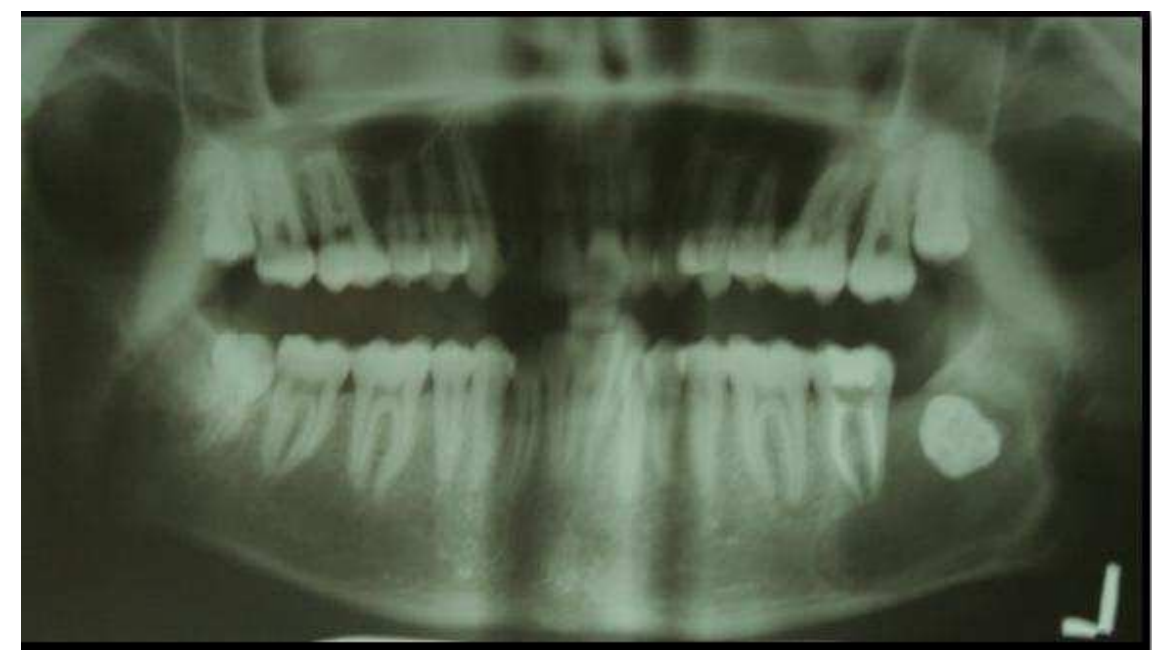

FIGURE 2 - Pre Operative OPG Showing Endodontic Treatment With 37 Which Was In Close Vicinity With The Lesion. 


\section{CASE REPORT}

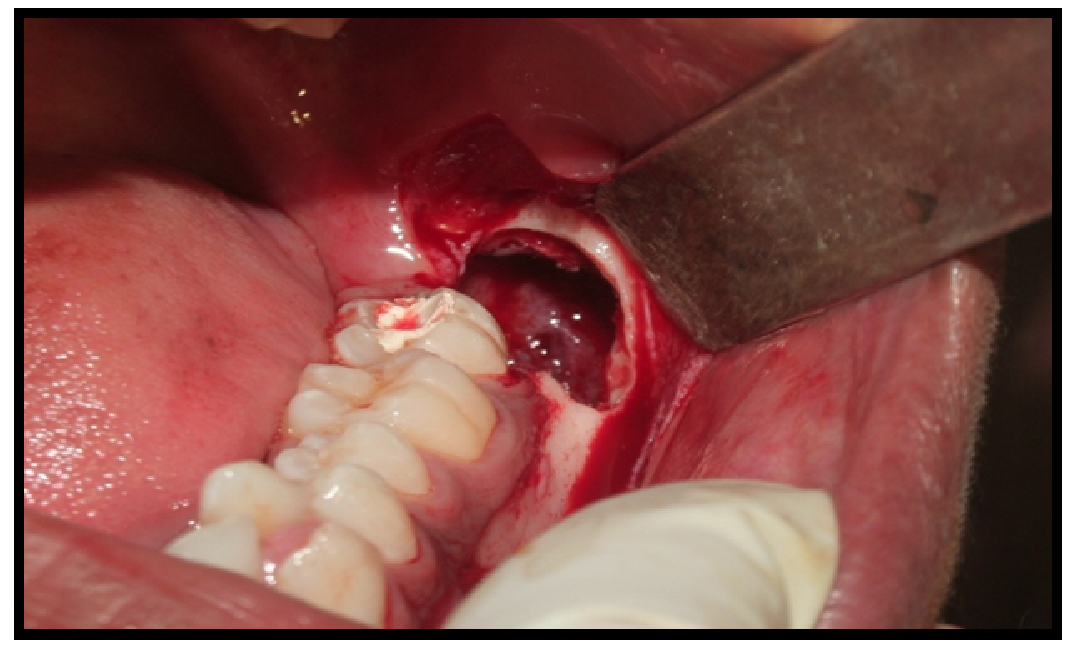

FIGURE 3 - Bony Window Creation \& Cystic Lining Identification Enveloped With Floating Left Third Molar.

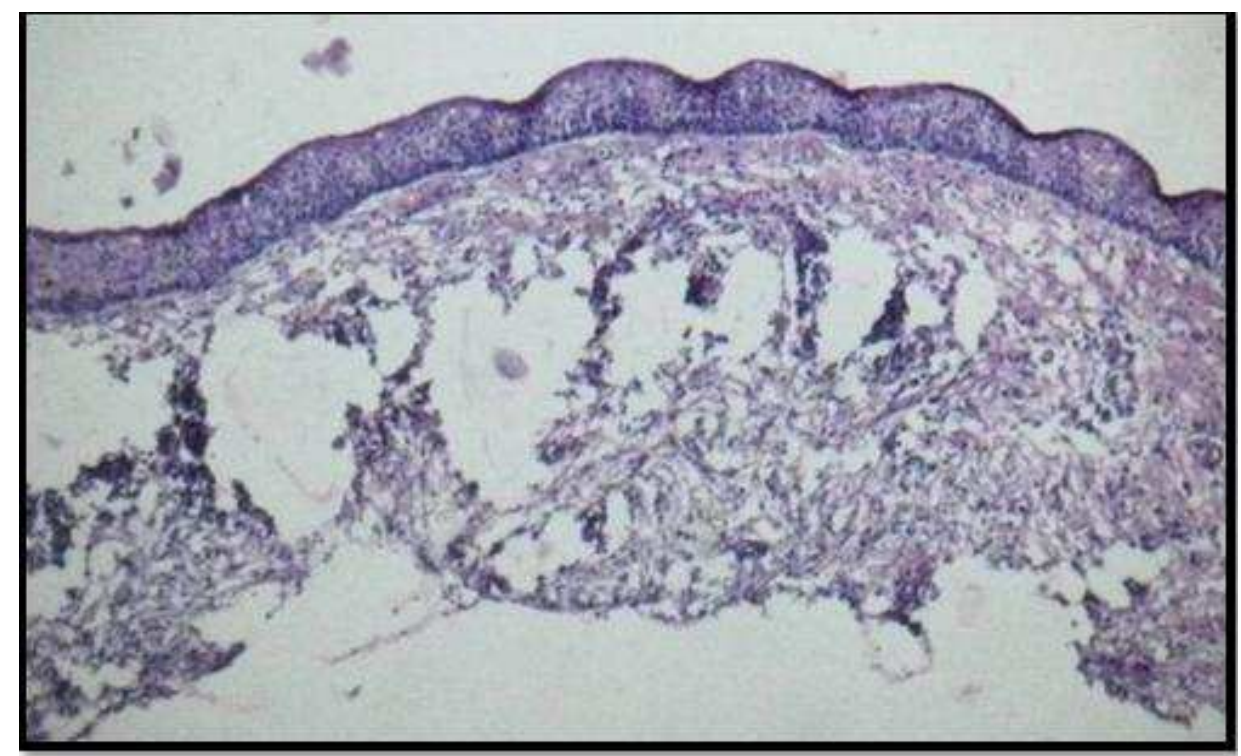

FIGURE 4 - Typical Histopathologic Appearance Of The KCOT Showing Parakeratinized Stratified Epithelium With Tombstone Appearance. (Haematoxylin \& Eosin stain). 